\title{
Accessories to Enhance Adenoma Detection Rates: Is the Endocuff Better than the Conventional Cap for Trainees?
}

\author{
Tae-Geun Gweon ${ }^{1}$ and Sang-Bum Kang ${ }^{2}$ \\ Division of Gastroenterology, Department of Internal Medicine, ${ }^{1}$ College of Medicine, The Catholic University of Korea, Seoul, ${ }^{2}$ Daejeon \\ St. Mary's Hospital, College of Medicine, The Catholic University of Korea, Daejeon, Korea
}

See "Endocuff-Assisted versus Cap-Assisted Colonoscopy Performed by Trainees: A Retrospective Study" by Yutaka Okagawa, Tetsuya Sumiyoshi, Yusuke Tomita, et al., on page 339-345.

Colonoscopy can prevent colorectal cancer (CRC) by detecting and removing precancerous lesions including colorectal adenomas. ${ }^{1}$ The adenoma detection rate (ADR), defined as the percentage of subjects with at least one adenoma detected during colonoscopy, is the most important quality indicator for colonoscopy as the ADR is inversely associated with the rate of post-colonoscopy CRC. ${ }^{2}$ An inspection time of at least 6 minutes and adequate bowel cleansing are associated with higher ADR. ${ }^{3}$ Devices such as a conventional cap, Endocuff, and Endoring, which are distally attached to the colonoscope, were developed to enhance the ADR. ${ }^{4}$ Distal attachment devices can flatten colon folds, thereby enhancing the visualization of concealed areas. Clinical trials using the conventional cap produced mixed results and could not demonstrate a beneficial effect on ADR. The Endocuff (Arc Medical Design Ltd., Leeds, England) is a novel distal attachment device comprising several soft, flexible plastic arms. A meta-analysis demonstrated an increased ADR for the Endocuff compared to that of standard colonoscopy; i.e., colonoscopy without any distal attachment device. ${ }^{4}$ Several studies comparing ADR between

Received: March 4, 2020 Accepted: March 26, 2020

Correspondence: Sang-Bum Kang

Division of Gastroenterology, Department of Internal Medicine, Daejeon St. Mary's Hospital, College of Medicine, The Catholic University of Korea, 64 Daeheung-ro, Jung-gu, Daejeon 34943, Korea

Tel: +82-42-220-9501, Fax: +82-42-252-6807,E-mail: dxandtx@catholic.ac.kr ORCID: https://orcid.org/0000-0002-1946-7896

(c) This is an Open Access article distributed under the terms of the Creative Commons Attribution Non-Commercial License (http://creativecommons.org/ licenses/by-nc/3.0) which permits unrestricted non-commercial use, distribution, and reproduction in any medium, provided the original work is properly cited. the Endocuff and conventional cap showed that the ADR with the Endocuff was not higher than that with the conventional cap. $^{5,6}$

In this issue of Clinical Endoscopy, Okagawa et al. report a comparison of ADR between the Endocuff and conventional cap. ${ }^{7}$ This single-center retrospective study included 94 patients in the Endocuff group and 193 patients in the conventional cap group. Colonoscopy was performed by three trainees. The demographic characteristics of the patients and technical colonoscopy parameters (cecal intubation rate, cecal intubation time, and withdrawal time) were comparable between the two groups. The ADR in the Endocuff group was significantly higher than that of the conventional cap group (Endocuff, 54.3\% vs. cap, 37.3\%, $p=0.019$ ). Other parameters related to polyp detection (mean number of polyps per patient, polyp detection rate) were also superior in the Endocuff group. Although the differences were not statistically significant, the ADR in the Endocuff group was higher than that in the cap group for all three colonoscopists.

The ADR is also influenced by physician-related factors. ${ }^{8}$ Several studies have suggested that the ADR of gastroenterologists, who are regarded as more skillful endoscopists, was higher than that of surgeons. ${ }^{8}$ The endoscopist's skill is an important factor in enhancing the $\mathrm{ADR}{ }^{3}$ A previous study showed that, despite a shorter inspection time, the ADR of experienced endoscopists was higher than that of beginners. ${ }^{9}$

The main result of this study differs from that of recent studies showing comparable ADRs between the Endocuff and conventional cap. ${ }^{5,6}$ The authors of this study did not determine why the Endocuff provided a superior ADR compared 
with the conventional cap in their study, the difference could be related to differing mechanisms between the conventional cap and the Endocuff. The conventional cap is "fixed" on the distal tip of the colonoscope and its shape is uniform regardless of bowel and colonoscope movement. When using the conventional cap, the colonoscope must closely approach the colon wall, which prevents visualization of the other side of the colon wall. In contrast, the flexible soft wings of the Endocuff are dynamically flattened or spread according to the movement of the colonoscope. Moreover, the Endocuff's wings are wider than those of the colonoscope, at an estimated $35 \mathrm{~mm}$ when fully spread. ${ }^{10}$ Therefore, the colonoscope can be centrally positioned during withdrawal, which can minimize the concealed areas of the colon.

The results of this study suggested that the Endocuff might be superior to the conventional cap for improving trainee ADR. The high workloads of attending physicians in teaching hospitals prevent them from providing expert guidance to all beginners performing colonoscopies. In this context, the results of this study provide useful information. The use of the Endocuff might increase trainee ADR and reduce their chances of missing adenoma.

However, this study has several limitations. This study single-center, retrospective study included a relatively small number of colonoscopists $(n=3)$ and colonoscopies $(n=287)$. Previous randomized controlled trials did not demonstrate the superiority of the Endocuff over the cap for ADR. ${ }^{5,6} \mathrm{Al}-$ though the results of the present study suggest the superiority of the Endocuff for trainees, the results are questionable. A previous study including two experienced and two trainee colonoscopists ${ }^{6}$ showed comparable ADRs for the Endocuff and conventional cap, although subgroup analysis according to colonoscopist experience was not performed. Therefore, well-designed studies are needed to validate the superiority of the Endocuff for trainees.
Conflicts of Interest

The authors have no financial conflicts of interest.

ORCID

Tae-Geun Gweon: https://orcid.org/0000-0002-0884-7228

\section{REFERENCES}

1. Zauber AG, Winawer SJ, O’Brien MJ, et al. Colonoscopic polypectomy and long-term prevention of colorectal-cancer deaths. N Engl J Med 2012;366:687-696.

2. Kaminski MF, Regula J, Kraszewska E, et al. Quality indicators for colonoscopy and the risk of interval cancer. N Engl J Med 2010;362:17951803.

3. Gupta N. How to improve your adenoma detection rate during colonoscopy. Gastroenterology 2016;151:1054-1057.

4. Facciorusso A, Del Prete V, Buccino RV, et al. Comparative efficacy of colonoscope distal attachment devices in increasing rates of adenoma detection: a network meta-analysis. Clin Gastroenterol Hepatol 2018;16:1209-1219.e9.

5. Sola-Vera J, Catalá L, Uceda F, et al. Cuff-assisted versus cap-assisted colonoscopy for adenoma detection: results of a randomized study. Endoscopy 2019;51:742-749.

6. Imaeda H, Yamaoka M, Ohgo H, et al. Randomized control trial of adenoma detection rate in Endocuff-assisted colonoscopy versus transparent hood-assisted colonoscopy. J Gastroenterol Hepatol 2019;34:14921496.

7. Okagawa Y, Sumiyoshi T, Tomita Y, et al. Endocuff-assisted versus cap-assisted colonoscopy performed by trainees: a retrospective study. Clin Endosc 2020;53:339-345.

8. Mehrotra A, Morris M, Gourevitch RA, et al. Physician characteristics associated with higher adenoma detection rate. Gastrointest Endosc 2018;87:778-786.e5.

9. Kim YD, Bae WK, Choi YH, et al. Difference in adenoma detection rates according to colonoscopic withdrawal times and the level of expertise. Korean J Gastroenterol 2014;64:278-283.

10. ASGE Technology Committee, Konda V, Chauhan SS, et al. Endoscopes and devices to improve colon polyp detection. Gastrointest Endosc 2015;81:1122-1129. 